\title{
Qualitative physics as a component in natural language semantics: A preliminary report
}

\author{
Sven E. Kuehne (skuehne@northwestern.edu) \\ Qualitative Reasoning Group, Northwestern University \\ 1890 Maple Avenue, Evanston, IL, 60201, USA \\ Kenneth D. Forbus (forbus@northwestern.edu) \\ Qualitative Reasoning Group, Northwestern University \\ 1890 Maple Avenue, Evanston, IL, 60201, USA
}

\begin{abstract}
We propose that qualitative physics can provide an important component of natural language semantics. Specifically, we describe how qualitative process theory can be recast in terms of frame semantics, as used in the Berkeley FrameNet project. This reformulation is important because it could allow the techniques of qualitative reasoning to be harnessed for natural language understanding and it expands the range of phenomena that can be described in NL semantics. We show that these ideas can account for a large percentage of a small corpus of explanatory text, and that they support the construction of QP models from such texts.
\end{abstract}

\section{Introduction}

Understanding the semantics of natural language is a central problem in cognitive science. Such an understanding must connect fundamentals of our conceptual structure to their realizations in linguistic forms, and thus must draw upon both insights about language and about conceptual structure. Significant progress is being made on the language side, with projects such as FrameNet (Fillmore et al 2001) developing broad systems that capture aspects of the meaning of words and linguistic constructions in terms of frame semantics (Fillmore \& Atkins, 1994). Significant progress is also being made on understanding aspects of human conceptual structure, for example, the work carried out in the qualitative reasoning community. Qualitative reasoning focuses on the representations necessary to reason about the physical world, ranging from everyday phenomena to the work of scientists and engineers. While many QR efforts are aimed at applications, some efforts are aimed directly at modeling human reasoning about physical systems (cf. Bredeweg \& Schut, 1991; Kuipers \& Kassirer 1984; Kuipers et al 1988; Forbus \& Gentner 1986, 1997). Ultimately these two lines of investigation, natural language semantics and the understanding of human conceptual structures, need to join forces. In the words of the FrameNet team": "In the end it will be necessary to express frame notions in some formal knowledge-representation language which will allow valid inferences to be drawn from frame semantic representations of sentences, or which

\footnotetext{
1 The FrameNet project's home is the International Computer Science Institute in Berkeley, CA. A detailed description of the project can be found at http://www.icsi.berkeley.edu/ framenet
}

can serve in a precise way in the development of a cumulative representation of the content of an ongoing discourse."

This paper is a first step in linking these investigations. We propose that qualitative process theory (Forbus 1984) can provide such a knowledge representation language for aspects of frame semantics concerned with continuous parameters and continuous causation. QP theory concerns the structure of a class of physical theories, and has been successfully used in a variety of reasoning systems (Forbus, 1996). The hypothesis is that many mental models of physical phenomena can be expressed in this formalism. QP theory has been used to develop a wide range of models of phenomena, including economic and medical models in addition to physical models. This makes it an excellent candidate for a component in a larger system of natural language semantics.

We begin by building a theoretical bridge between QP theory and frame semantics, as exemplified in FrameNet. We briefly review the relevant aspects of QP theory and show how they can be recast in terms of frame semantics. This recasting provides a means for defining frames for physical processes and relationships involving continuous parameters. We illustrate how these ideas can be used to extend a natural language semantics by an analysis of flow.

With the theoretical bridge in place, we provide empirical support for it via a corpus analysis of an explanatory text. This analysis provides evidence concerning two questions. First, we explore how much of the explanation these frames can account for. Second, we analyze whether a qualitative model can be reconstructed from the text using these frames. Finally, we discuss some new issues raised by this approach and plans for future work.

\section{Qualitative physics in frame semantics}

We begin by recasting QP theory in terms of frame semantics, as used in FrameNet. We review the ontological assumptions underlying QP theory and their implications for the organization of the frame system. Next we discuss physical processes and their occurrences, followed by an analysis of how qualitative mathematics is expressed. We conclude this section by showing that this analysis is compatible with analyses of overlapping phenomena already in FrameNet. 


\section{Ontological assumptions}

In QP theory, physical changes in continuous properties are caused by physical processes. Examples of physical processes include kinds of flows (e.g., heat, liquid, gas), phase changes (boiling, freezing), and some aspects of motion. Ontologically, physical processes serve as the mechanisms of physical causality: All naturally occurring changes (and many of the indirect effects of the actions of agents) are ultimately caused by the activity of one or more physical processes. Instances of physical processes exist when an appropriate configuration of participants occurs. Such process instances are active over any span of time for which their conditions hold. When a process instance is active, its consequences hold. For example, two thermal entities (i.e., having the continuous property heat) that are thermally in contact give rise to two instances of heat flow, one in each potential direction. Whether or not either of these is active depends in turn on the relative temperatures between the two bodies.

The consequences of a physical process are of three types. First, there are direct influences that represent the direct effects that a physical process has on the world. For example, heat flow causes the heat of the source of the flow to decrease while increasing the heat of the destination. Second, there are other dynamical properties defined, including new parameters and causal laws, which describe how changes propagate through continuous properties. For example, the rate at which heat flows is a continuous property, and it is determined by the difference between the temperatures. Third, other properties that hold while the process is occurring, such as appearance information, can be consequences. In everyday boiling, for instance, one typically sees bubbles.

Two key conceptual advances in qualitative modeling are the insights that (1) many important kinds of reasoning about dynamical systems can be done without numerical information or mathematical models and (2) qualitative relationships can be formulated which explicitly capture patterns of human causal reasoning. These causal connectives are summarized below; see (Forbus 1984) for details. The values of continuous parameters tend to be expressed in comparative terms, via ordinal relationships constraining a parameter with respect to other relevant properties. If an object participates in process instances of heat flow, for example, then its temperature is defined in terms of its relationships with the temperatures of the other objects participating in those heat flows.

This summary highlights three important properties of QP theory that makes it potentially a valuable component of natural language semantics. First, the notion of physical process it defines is psychologically plausible. Descriptions of physical processes are abundant in language concerning physical phenomena, and are routinely used in metaphors (cf. Lakeoff 1980, Gentner et al 2001). Second, the causal account QP theory provides is consistent with human causal explanations in most physical domains (Forbus \& Gentner 1986, 1997). Third, the abstract level of information that qualitative representations support seems a natural fit for the level of specificity commonly found in natural language descriptions of physical principles and situations. One does not need to understand differential equations or carry out detailed simulations to understand physical metaphors ("her anger mounted until she boiled over").

\section{Frame Semantics}

In frame semantics, meaning is expressed in terms of systems of structured representations, frames, which provide the links between words and conceptual structures. Lexical items are linked to frames as such that they highlight a particular frame. For example, the occurrence of the noun 'growth' might evoke the Expansion frame in the Space domain, while the verb 'push' activates the cause-to-move frame in the Motion domain.

The participants, props, and other conceptual roles involved in a frame are called frame elements (FE). FEs are linked to parts of a text and have associated with them inferences that provide meaning (Fillmore \& Atkins, 1994). For example, the cause-to-move frame in the motion domain includes frame elements for the agent, the theme (i.e. the object acted on), the source, the path, the goal (i.e. the destination) and many more. Not all FEs of a frame are always present, many of them are optional and are used in combinations to express specific grammatical realizations of a frame. Although frame elements are similar to thematic roles and case roles, it is important to note that frame semantics does not define a universal set of possible frame elements. Frame elements are specific to the frame they are defined in, and more FEs can be added to a frame if the underlying grammatical structures require them.

Frames can range from simple patterns and states to highly complex scenarios. Scenarios consist of several scenes and transition states (both frames themselves) and information about their temporal ordering and occurrence.

The packaging of physical knowledge and principles in QP theory (inspired in part by Minsky's (1975) notion of frames) suggests a natural alignment with frame semantics. There is a basic physical process frame, whose structure provides the fundamental aspects of physical processes. Subframes are elaborations of this frame and describe particular categories of physical processes, with differences in their participants and consequences being the differentia that set them apart. A subframe inherits all the FEs of its parent frame and might add several new ones. For example, in our formalism below, the Conduction-Heatflow frame is a subframe of the Heatflow frame, which itself is a subframe of the Physical-Process frame. All frame elements of the Physical-Process frame are available in the ConductionHeatflow frame. Instances of these frames are combined with frames from other aspects of the semantics to create the frame system describing the meaning of a text. FrameNet provides support for multiple inheritance, frame blending (i.e. the simultaneous activation of two frames), and frame composition (i.e. the definition of scenario as a sequence of scenes) (cf. Johnson et al, 2001; Fillmore et al, 2001). 
The qualitative causal mathematics of QP theory is expressed through another collection of frames. In addition to their role in physical process descriptions, these qualitative causal frames can be used for other domains with continuous parameters, such as economics or metaphorical extensions of physical concepts. The next three subsections outline these frame systems.

\section{Processes and their occurrences}

The Physicalprocess frame involves four types of FEs:

- Participant specifies one of the participants in the physical process. Example: in "Heat flows from the hot brick to the cool room", "hot brick" and "cool room" are Participants in an instance of the HeatFlow frame.

- Condition specifies one of the conditions under which the process is active. Example: in "Heat flows from one place to another because the temperature of the two places is different." the condition is the difference in temperature values (see ordinals below)

- Status specifies whether or not the process is active. Example: In "The radiator leak was stemmed by shoving a cloth into it." The word stemmed suggests that a flow which was enabled is now stopped. We say that the status is active when the process is occurring, and inactive otherwise.

- Consequence specifies one of the direct consequences of the physical process. Example: In "Water flooded into the room when the valve broke." the liquid flow into the room has as one of its consequences an increase in the amount of water in the room.

These frame elements can be directly mapped to the formal models that QP theory supports. For a process type or instance, the set of participants collectively define the collections of entities it occurs among. The union of the conditions is the set of conjuncts that comprise the necessary and sufficient conditions for it to be active. The set of fillers for the consequences FEs constitute its direct consequences.

Our analysis of the syntactic realizations of these frame elements, and the others reported here, is work in progress, and we plan to analyze a much larger corpus to ensure that our results are robust. That, plus space limitations, will limit our discussion of syntactic realizations to a few stable highlights. Noun phrases that serve as the primary actor and object in a sentence tend to be participants, e.g., in "A hot brick loses heat to a cool room." "Hot brick" and "cool room" are participants. Certain frame elements already used in other FrameNet frames, e.g., Source and Destination, tend to be participants when a physical process is the actor. The patterns that indicate conditions include "Condition causes Process", "Process occurs when condition.", and "Process depends on Condition." For consequences, there are two cases: influences and other consequences. Influences are discussed below. The other consequences, since they can range over almost any physical statement in principle (e.g., appearances, sounds, etc.), are difficult to characterize concisely. Example indicators are occurrences of the FrameNet FEs of Manner and Result.

\section{Parameters and values}

Continuous properties are represented by the Quantity frame, which has the following elements:

- Entity specifies what this property is a property of. Typically this is unique. Example: "brick" in "the temperature of the brick"

- QuantityType specifies the kind of parameter that this is. Example: "temperature" in "temperature of the brick."

- Value specifies the numerical value of the property. This FE is optional. Example: " 3 " in "3 liters of water".

- Units specifies the physical units of the property. This FE is optional. Example: "kilograms" in "3 kilograms of lead".

Ds specifies how the parameter is changing and stands for "sign of the derivative". This FE is optional. Example: In "The temperature is increasing." the sign is expressed by the word "increasing" which would be mapped to the value of 1. While syntactic realizations for quantity types, values and units are fairly obvious, Ds manifests itself in the text many different ways, e.g. -1 could show up as "falling", "decreasing", etc.

Values and units are often not explicitly stated or even filled in via default, but Ds and comparative statements about values are common. These are expressed via the Ordinal frame, which has the following FEs:

- $Q 1, Q 2$ specify the quantities being compared. Either is optional. Example: "the coffee's temperature".

- OrdReln specifies the relationship between the values of the quantities. It must be one of $<,>,=, \geq, \leq,=. \neq$, sameorder, or negligible. Example: In "Evaporation can be ignored" the word "ignored" refers to a negligible ordReln of the rate of an evaporation process compared to other processes being described.

Ordinal relations provide a useful qualitative notion of value because they often serve as conditions for physical processes and states (e.g., flows occur when a driving parameter is unequal, equilibriums occur when opposing effects are equal). Syntactic realizations of ordinals are usually described via explicit comparisons (e.g., "Q1 is greater than $\mathrm{Q}^{2}$ ") or as some type of comparative construction. One very common pattern is the use of ordered dimensional adjectives to set up a tacit comparison. For instance, from "hot brick" and "cool room", one knows an ordinal relationship involving their temperature due to the meanings of "hot" and "cool".

\section{Qualitative mathematics and causality}

The causal relationships between quantities are expressed via a qualitative mathematics that supports partial information about the nature of the connections between them. The basic frame is the Influence, whose FEs are

- Constrained specifies the dependent quantity, i.e., the effect. 
- Constrainer specifies the independent quantity, i.e., a proximal cause for the constrained quantity.

- Sign specifies the direction, which can be + or - . It is expressed by words such as "up", "down", "greater", "more", "less" etc.

There are two subframes of the Influence frame, DirectInfluence and eprop. These correspond to the QP theory primitives $\mathrm{I}+/ \mathrm{I}-$ and $\propto_{\mathrm{Q}^{+}} / \propto_{\mathrm{Q} \text { - }}$ respectively (Forbus 1984). While the two subframes share frame elements, the underlying semantics is quite different. For direct influences, the constrainer is combined via addition to other constrainers to determine (qualitatively) the derivative of the constrained quantity, and the sign indicates whether it is a positive or negative contribution to that sum. For eprop, the constrained is functionally dependent on the Constrainer, and perhaps on other properties as well, with the sign indicating whether the dependence is increasing or decreasing monotonic. This is the weakest distinction that enables changes to be propagated through causal laws.

As their common heritage suggests, in some cases the syntactic realizations of these two kinds of influences can be quite close. However, many cases are straightforward. Some realizations for eprop include "Constrained depends on Constrainer." and "As Constrainer Ds, Constrained Ds." For example, "As the air temperature goes up, the relative humidity goes down" is clearly a eprop, with Constrained = "relative humidity", Constrainer = "air temperature", and Sign = -.

Syntactic realizations for DirectInfluences are more complex. In advanced texts one can find patterns such as "The rate of constrained depends on Constrainer." but they do not seem common. In everyday texts explicit discussions of rates seem even rarer. Instead, DirectInfluences tend to occur in larger-scale patterns, often tied to a generalized notion of motion. For example, "Most water in the air comes from evaporation." is a DirectInfluence, with Constrained = "water in the air", Constrainer $=$ "[rate of] evaporation" and sign $=+.{ }^{2}$

\section{Compatibility with existing frame semantics}

One implication for semantics is that, in addition to the frames associated with QP theory per se, there will be a collection of subframes corresponding to particular kinds of physical phenomena, such as flows, motion, and phase changes. And indeed FrameNet already has an existing analysis of motion that is compatible with QP theory. The FEs of Theme, Source, Goal, and Path are finer-grained distinctions of the FE Participant in the general Physicalprocess frame. In QP theory models of this kind of motion (cf. Forbus 1984), there is a quantity Position that is referenced to the Path from Source to Goal. A DirectInfluence frame with Constrained $=$ Position and

\footnotetext{
${ }^{2}$ Note that 'from evaporation' refers to an internal quantity of a process (i.e. the evaporation rate), not to a participant (as in 'from the ocean'). The latter would be marked as a source, not as a constrainer.
}

Constrainer $=$ Velocity is a Consequence of the Motion frame. An ordinal frame with $\mathrm{Q} 1=$ velocity, $\mathrm{Q} 2=$ zero, and ordReln $=\neq$ is the Condition for the Motion frame. This compatibility is encouraging, since it means that the implications that can be drawn from qualitative reasoning could be made available in service of natural language understanding.

\section{Example: An analysis of flow}

Next we present an analysis of an important frame for physical phenomena, flow. We start with well-worked out ideas in the qualitative physics literature, using the framework above to recast them into frame semantics. This frame is used in our corpus analysis below.

\section{The general Flow frame}

The model of flow we are starting with is based on those in Forbus (1984). Several of the frame elements are specializations of Participant:

- FlowSource specifies the starting region of the flow.

- FlowDest specifies the region where what is flowing ends up.

- FlowPath specifies the path along which the flow occurs

These FEs determine the overall type of flow occurring:

- FlowDriver specifies the intensive quantity (e.g., something like pressure or temperature) whose difference at source and destination drives the flow.

- Flow $Q$ specifies the extensive quantity (e.g., something like mass or heat) that is directly influenced by the flow. Optional.

- FlowStuff specifies the "stuff" which is considered to be flowing. Optional.

Typically texts mention either Flowe or Flowstuff but not both. Many uses of the Flow frame are metaphorical from a scientific perspective (e.g., heat is not a substance), but may be literal from a common sense perspective, depending on the language user's mental models. Flowstuff must be continuous in nature (hence $\mathrm{FlowQ}$ must exist, even if not explicitly mentioned) for the idea of flow to make sense.

There are two condition FEs for Flow. The first is an Ordinal, i.e., that the FlowQ (FlowSource ( $F l o w)$ ) is greater than FlowQ (FlowDest (Flow)). The second is that FlowPath not be blocked. The nature of being blocked depends on the subframe of Flow. For instance, a stopper can block liquid flow, but heat can still pass.

Flow has three consequence FEs: a Quantity whose QuantityType is Rate, and two DirectInfluence frames, constraining the Flowe of Flowsource and FlowDest via Rate (Flow) with the appropriate signs.

More sophisticated versions of this basic pattern are common in qualitative modeling; e.g., the rate is typically a function of the difference between the driving quantities, and also depends on path properties. Such elaborations do commonly appear in explanatory texts, and consequently the ability of qualitative modeling to support such incremental 
elaboration, which can be done via additional eprop frames in this case, provides a necessary source of representational flexibility for natural language semantics.

\section{Some empirical evidence}

Up to now we have been concerned with expressing the concepts of QP theory in Frame Semantics, showing how they can fit into this larger system and some of their syntactic realizations in English. Here we examine the utility of doing this, in two ways. First, we turn the question around: How much do these ideas contribute to understanding the semantics of texts involving the physical world? Second, can we use these ideas to reconstruct from an explanatory text the physical ideas being communicated? We examine each in turn.

How far can a QP-based frame semantics go in accounting for the semantics of explanatory texts? One way to answer this question is to analyze a corpus of physical explanations, and see what fraction of the sentences require the frames of QP theory (and frames for mental models expressed in QP theory) for their interpretation. We have done this by using four chapters of a book on solar energy, Sun Up to Sun Down (Buckley, 1979). We chose this book because it is very clearly written, and we have been using it for a source of examples in other projects, since it uses both diagrams and analogies heavily. ${ }^{3}$ We chose chapters 2 through 5 because they provide a basic exposition of heat, temperature, and types of heat flow.

Our analysis method was this. Two evaluators familiar with the theory independently scored each sentence. Then they compared their results, discussing divergences until they came to agreement.

We looked at the linguistic realizations of physical processes in the text. Based on the QP frame semantics, we defined nine types of information about processes: the process name $(\mathrm{P})$, information about subclasses of a process (i.e. a specialization) (SC), participants (PA), about conditions: antecedent activations (AA), antecedent ordinal relations (AO), antecedent relations (AR), and finally about consequences: indirect influences (CII), direct influences (CDI), and consequence relations other than influences (CR). ${ }^{4}$ Multiple pieces of information can appear within a single sentence, so we scored number of phrases of particular types in addition to the number of sentences that they occurred in. Sentences can contain multiple types of information, so the same sentence can appear in multiple categories. We also distinguished between information from examples (identified through a preliminary analysis)

\footnotetext{
3 As additional corpus material, we have selected a college textbook on the weather as well as a children's book on weather, but while these have served as a source of data for our analysis of syntactic realizations, the results in this section are based on the Buckley text.

4 Although the information involved in these categories has varying complexity, e.g., influences and ordinal relations are more complex than the process or participant names, we do not impose any ordering or weighting on these pieces of information.
}

and general information, since we have hypothesized (Forbus \& Gentner, 1997) that common sense physics arises from within-domain analogies involving concrete descriptions. Tables 1 (general information) and 2 (exemplar-specific information) show our results.

\begin{tabular}{|l|l|l|l|l|l|l|l|l|l|}
\hline Type & P & SC & PA & AA & AO & AR & CR & CDI & CII \\
\hline \#Sentences & 10 & 1 & 8 & 15 & 5 & 1 & 9 & 8 & 15 \\
\hline \#Phrases & 11 & 4 & 14 & 16 & 5 & 1 & 18 & 16 & 18 \\
\hline
\end{tabular}

Table 1: General statements using QP theory concepts

\begin{tabular}{|l|l|l|l|l|l|l|l|l|l|}
\hline Type & P & SC & PA & AA & AO & AR & CR & CDI & CII \\
\hline \#Sentences & 26 & 0 & 28 & 15 & 6 & 5 & 26 & 19 & 14 \\
\hline \#Phrases & 26 & 0 & 74 & 15 & 7 & 5 & 53 & 38 & 17 \\
\hline
\end{tabular}

Table 2: Use of QP theory concepts in examples.

The data shows that the exemplar-specific data contains more than twice the number of processes, about five times the number of participants and a lot more information about the consequences of the mentioned processes. ${ }^{5}$ However, the amount of information about the conditions of a process (categories AA, AO, and AR) is nearly the same. As expected, any information about specialization of processes (SC) is only found in the general information.

What kind of coverage does QP theory provide? Of the 216 sentences, 94 of them mention at least one element from the QP frame system proposed here. That means that QP theory can account for roughly $43 \%$ of these chapters.

Let us turn to the second question, the reconstruction of a QP domain theory from the frame semantics that one might get from analyzing a text. Again we rely on Chapters 2 through 5 of Buckley (1979). These chapters yield six physical processes: General models of heat flow and volume flow (e.g., liquid flow), and four subclasses of heat flow (conduction, convection, radiation, and transport). Using this data and the information about the constituents of processes it contains, we attempted to manually reconstruct the models of the underlying physical processes. Figure 1 shows the reconstructed model of the generic heat flow process. One piece of information in Figure 1 marked with a star was not part of any general description of the heat flow process but originated from information about specific examples. It was generalized and included into the generic process model. By combining information from specific examples with general information, reasonable QP theory process descriptions of each were obtained.

These results are very encouraging. The frame semantics based on QP theory provides significant coverage of this corpus. The aspects that are not related to QP theory are not themselves physical laws or behaviors per se, but require frames of the types that would be found in other kinds of texts. Thus these results suggest that the frame semantics

\footnotetext{
${ }^{5}$ The ratio between general and exemplar-specific information is an effect of the popular-science nature of the source text. It also illustrates the point that we need to incorporate mechanisms for abstracting general knowledge from concrete exemplars.
} 


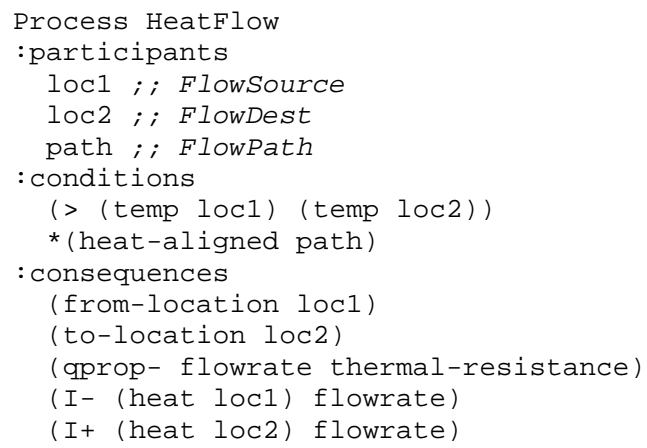

Figure 1: One QP physical process description reconstructed from the Buckley text. The line marked with "*" was derived via generalization from specific examples.

we propose using QP theory could play a useful role in a broad system of natural language semantics.

\section{Discussion}

This paper argues that qualitative physics, specifically QP theory, can be used as a component in a system of natural language semantics. We outlined how QP theory can be recast in terms of Fillmore's frame semantics, as used in the FrameNet project. The constructs of QP theory can be recast in terms of a collection of frames and subframes, which can be used to describe many causal mental models found in explanatory texts. As the syntactic realizations of these frames are further worked out, we believe that they will be a valuable extension to FrameNet semantics.

In addition to broadening the coverage of FrameNet to include a wide range of continuous phenomena and systems, our extension also grounds these new frames in terms of a well-worked out knowledge representation formalism capable of supporting qualitative reasoning. The compatibility of existing FrameNet motion descriptions with this model, and our analysis of a QP model of flow in frame semantics, lends support to our claim that this recasting of qualitative modeling can productively extend frame semantics for natural language.

The corpus analysis presented suggests that this extension can be useful, since $43 \%$ of the material in sample chapters from a typical science book can be captured in terms of them. Moreover, our analysis suggests that these frames could be composed to construct domain theories of a kind already used in qualitative reasoning.

Our results suggest that QP frame semantics can indeed play an important role in natural language semantics for physical texts. More investigation is needed on several questions, including:

- We want to refine our estimates of coverage by analyzing a larger corpus with a broader range of materials. These analyses are impractical by hand, so we are exploring the use of automated tools for subsequent analyses.
- We view our work as complementary to that of Narayanan (1999), who is linking FrameNet semantics with sensory-motor schemata. Both will ultimately be needed, and their interplay will be interesting to explore.

- $\quad$ The same QP analysis used for literal language could be used to improve the productive understanding of many metaphors. For example, the FrameNet analysis of heat in the emotional domain has tied to it the lemma boil directly. If QP representations for heat, heat flow, and boiling were used instead, one could infer that making someone angry for longer could lead to boiling, and that if someone had "boiled over", starting a heat flow with them as the source, could "cool them off".

- A fascinating set of questions arises from crosslinguistic comparisons. Are these ideas bundled up in the same way in all languages, or are they realized very differently (e.g., Bowerman's (1996) cross-linguistic analysis of spatial prepositions, Talmy's (1985) crosslinguistic analysis of verb semantics, and Imai and Gentner's (1993) analysis of the mass/count distinction)?

Another goal of our analysis is synthesis, i.e., to create a habitable controlled language that can be used in natural language processing systems that communicate with people about the physical world more fluently. Such software could be invaluable in creating new kinds of intelligent software, such as tutoring systems and monitoring systems.

\section{Acknowledgments}

We thank Jason Trost for help with the corpus analysis, and Larry Birnbaum and Dedre Gentner for insightful comments on the paper. This work was supported by the Artificial Intelligence program of the Office of Naval Research.

\section{References}

Bowerman, M. (1996). Learning how to structure space for language: A cross-linguistic perspective. In $\mathrm{P}$. Bloom, M.A. Peterson, L. Hadel, \& M.F. Garrett (eds.), Language and space. Cambridge, MA: MIT Press, 385-436.

Bredeweg, B. \& Schut, C. (1991). Cognitive plausibility of a conceptual framework for modeling problem solving expertise. Proceedings of the $13^{\text {th }}$ Annual Conference of the Cognitive Science Society. Hillsdale, New Jersey: Lawrence Erlbaum, 473-479.

Buckley, S. (1979). Sun up to sun down. New York: McGraw-Hill.

Falkenhainer, B. \& Forbus, K. (1991). Compositional Modeling: Finding the Right Model for the Job, Artificial Intelligence, 51 (1-3).

Fillmore, C. J. \& Atkins, B. T. S. (1994). Starting where the dictionaries stop: The challenge for computational lexicography, In Atkins, B. T. S. and A. Zampolli (eds.) Computational Approaches to the Lexicon. Clarendon Press. 
Fillmore, C. J., Wooters, C. \& Baker, C. F. (2001). Building a Large Lexical Databank Which Provides Deep Semantics. Proceedings of the Pacific Asian Conference on Language, Information and Computation. Hong Kong.

Forbus, K. (1984). Qualitative Process Theory Artificial Intelligence, (24): 85-168.

Forbus, K. (1996). Qualitative Reasoning. CRC Handbook of Computer Science and Engineering. CRC Press.

Forbus, K. \& Gentner, D. (1986). Causal reasoning about quantities, Proceedings of the Eighth annual conference of the Cognitive Science Society, Amherst, Mass., August, 1986.

Forbus, K. \& Gentner, D. (1997). Qualitative mental models: Simulations or memories? Proceedings of the Eleventh International Workshop on Qualitative Reasoning, Cortona, Italy.

Gentner, D., Bowdle, B., Wolff, P., \& Boronat, C. (2001). Metaphor is like analogy. In D. Gentner, K. J. Holyoak, \& B. N. Kokinov (eds.) The analogical mind: Perspectives from cognitive science. Cambridge, MA: MIT Press, 199253

Imai, M., \& Gentner, D. (1993). Linguistic relativity vs. universal ontology: Cross-linguistic studies of the object/substance distinction. Proceedings of the Chicago Linguistic Society.

Johnson, C. R., Fillmore, C. J., Wood, E .J., Ruppenhofer, J., Urban, M., Petruck, M. R. L., Baker, C. F. (2001). The FrameNet Project: Tools for Lexicon Building. Manuscript, Version 0.7. Berkeley, CA.

Kuipers, B. J. \& Kassirer, J. P. (1984). Causal reasoning in medicine: analysis of a protocol. Cognitive Science 8: 363-385.

Kuipers, B. J., Moskowitz, A. J. \& Kassirer, J. P. (1988). Critical decisions under uncertainty: representation and structure. Cognitive Science 12: 177-210, 1988.

Lakeoff, G. \& Johnson, M. (1980). Metaphors we live by. Chicago: University of Chicago Press.

Minsky, M. (1975). A framework for representing knowledge. In: P. Winston (ed.) The Psychology of Computer Vision. New York: McGraw Hill: 211-7.

Narayanan, S. (1999). Moving Right Along: A Computational Model of Metaphoric Reasoning about Events. Proceedings of the National Conference on Artificial Intelligence (AAAI '99), Orlando, Florida, July 18-22, 1999, AAAI Press: 121-128.

Talmy, L. (1985). Lexicalization patterns: Semantic structure in lexical form, In T. Shopen (ed.), Grammatical categories and the lexicon, Vol. 3 of Language typology and syntactic description, CUP, Cambridge 